\title{
CARRIER COLLECTION INTO InGaAs/GaAs QUANTUM WELL: ROLE OF SURFACE BAND BENDING
}

\author{
G. Ambrazevičıus, S. Marcinkevičıus, T. Lideikis and K. Naudžius \\ Semiconductor Physics Institute, Goštauto 11, 2600 Vilnius, Lithuania
}

\begin{abstract}
Photoluminescence and photoluminescence excitation spectra of InGaAs/GaAs quantum wells located at different distances from a surface have been studied at $2 \mathrm{~K}$. The influence of surface band bending on carrier transfer into a quantum well is demonstrated. Oscillations due to relaxation of photo-excited carriers in GaAs barrier have been observed in the quantum well photoluminescence excitation spectra.
\end{abstract}

PACS numbers: $72.80 . \mathrm{Ey}, 73.50 . \mathrm{Gr}$

\section{Introduction}

The transfer of non-equilibrium carriers into a quantum well (QW) is of great significance for microstructure devices as well as for the physics of two-dimensional carriers. These processes, however, are still not completely understood. A wide range of experimental transfer time values (from $0.3 \mathrm{ps}$ [1] to $2 \mathrm{~ns}$ [2]) results in different conclusions concerning the transfer mechanisms.

We present the results of photoluminescence (PL) and PL excitation (PLE) studies in InGaAs/GaAs QW structures evidencing considerable role of surface electric field on carrier collection into the QW.

\section{Experimental}

Investigated QW structures grown by metalorganic chemical vapour deposition (MOCVD) on semi-insulating (SI) GaAs substrates, consisted of a $0.3 \mu \mathrm{m}$ GaAs buffer layer followed by an $\operatorname{In}_{0.18} \mathrm{Ga}_{0.82} \mathrm{As} 100 \AA \mathrm{QW}$ and a cap GaAs layer. The cap layer of initial $2 . \mu \mathrm{m}$ thickness was gradually etched to have the samples with the QW situated at different distances from the surface. The samples were not intentionally doped and had a background hole concentration of $3 \times 10^{15} \mathrm{~cm}^{-3}$. The PL and PLE speetra were measured at $2 \mathrm{~K}$ under the excitation of a halogen lamp with a $0.25 \mathrm{~m}$ scanning monochromator with $2 \mathrm{~nm}$ resolution. 


\section{Results and discussion}

The PL spectra of the investigated structures (Fig. 1) consist of two groups of PL lines: those related to the transitions $11 \mathrm{HH}$ between the electron and heavy hole ground states in the QW and to recombination in the GaAs barrier. Figure 1 shows that the QW PL line $11 \mathrm{HH}$ shifts towards red and strongly increases comparing to the PL lines of the GaAs barrier when the cap layer thickness is reduced from $2 \mu \mathrm{m}$ (A sample) to $0.2 \mu \mathrm{m}$ (B sample).

We attribute these features to the influence of the surface electric field: the quantum-confined Stark effect induced $11 \mathrm{HH}$ lineshift and drift enhanced photo-excited electron collection into the QW. The magnitude of the field $\varepsilon=$ $6 \times 10^{4} \mathrm{~V} \mathrm{~cm}^{-1}$, estimated according to [3] from the experimental value of the $11 \mathrm{HH}$ lineshift of $8 \mathrm{meV}$, satisfactorily agrees with the value $4 \times 10^{4} \mathrm{~V} \mathrm{~cm}^{-1}$, given by Schottky model (with $p=3 \times 10^{15} \mathrm{~cm}^{-3}$ and the surface band bending $0.6 \mathrm{eV}[4])$.

The comparison of integrated intensities of the QW and the barrier PL lines according to [5] gives the electron transfer times into the $\mathrm{QW}: \tau_{\mathrm{tr}}=10^{-9} \mathrm{~s}$ and $\tau_{\mathrm{tr}}=10^{-12} \mathrm{~s}$ for the samples $\mathrm{A}$ and $\mathrm{B}$, respectively. The difference in $\tau_{\mathrm{tr}}$ we associate with the drift in the surface electric field: the field aids electron transfer into the QW at the surface (B sample), but does not in the A sample with a thicker cap layer.
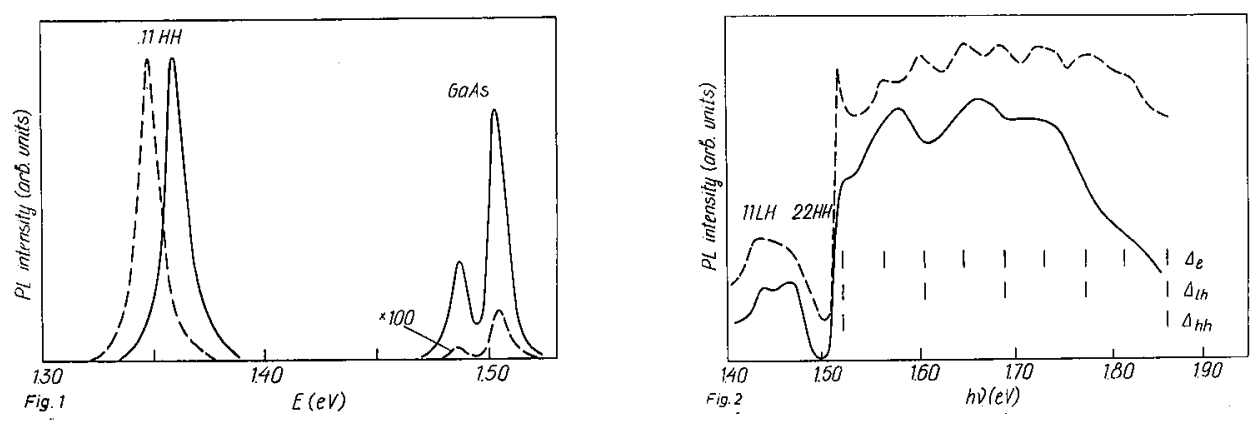

Fig. 1. $2 \mathrm{~K}$ photoluminescence spectra of the samples $\mathrm{A}$ (full curve) and $\mathrm{B}$ (broken curve) with a cap layer thickness being equal to $2.0 \mu \mathrm{m}$ and $0.2 \mu \mathrm{m}$, respectively. The spectra are normalized to the amplitude of the QW PL line.

Fig. 2. $2 \mathrm{~K}$ photoluminescence excitation spectra for the samples A (full curve) and B (broken curve). The oscillation periods $\Delta_{\mathrm{e}}, \Delta_{\mathrm{lh}}, \Delta_{\mathrm{hh}}$ are defined by Eqs. (1) and (2). The notations $11 \mathrm{LH}$ and $22 \mathrm{HH}$ mark transitions between the ground levels of electron and light-hole and between the first excited electron and heavy-hole levels in the QW, respectively.

The fast electron transfer is demonstrated in the QW PLE spectrum of the B sample (Fig. 2, broken curve). The spectrum displays oscillations [6] with the period

$$
\Delta_{\mathrm{e}}=\hbar \Omega_{\mathrm{LO}}\left(1+m_{\mathrm{e}} / m_{\mathrm{hh}}\right)
$$


where $\hbar \Omega_{\mathrm{LO}}=36.4 \mathrm{meV}$ is the energy of GaAs LO phonon, and $m_{\mathrm{e}}=0.067$ and $m_{\mathrm{hh}}=0.45$ are the electron and heavy-hole effective masses, respectively. As long as $\tau_{\mathrm{op}}<\tau_{\mathrm{tr}}<\tau_{\mathrm{ee}}$, where $\tau_{\mathrm{op}}$ and $\tau_{\mathrm{ee}}$ are the characteristic times of the electron-optical phonon and electron-electron interaction, respectively, the energy of electrons, approaching the QW, oscillates with the amplitude $\hbar \Omega_{\text {LO }}$ when the photo-excitation energy $h \nu$ is changed. Then, the energy dependence of the non-thermalized electron collection efficiency [6] and/or the electron capture rate [7] will cause the oscillations in the QW PLE spectra.

For the excitation densities used in our experiment $\tau_{\mathrm{ee}} \approx 10^{-10} \mathrm{~s}[8]$ and is longer than $\tau_{\mathrm{tr}}$ in the $\mathrm{B}$ sample. Additional photo-pumping shortens $\tau_{\mathrm{ee}}$ and the oscillations disappear in the PLE spectrum.

The electron transfer time in the A sample becomes comparable with the electron radiative lifetime in $\mathrm{GaAs}$ and recombination in the barrier appears to be a competing process of comparable intensity to the QW PL. Assuming the maximum recombination probability to be realized at $k=0$ we should expect the reduction of QW PL intensity at the photo-excitation energies $h \nu$ corresponding to the fast return of photo-excited carriers towards $k=0$. For carrier relaxation by emission of LO phonons these energies are

$$
h \nu=E_{\mathrm{g}}+n \Delta_{\mathrm{e}(\mathrm{lh}, \mathrm{hh})}
$$

where

$$
\begin{aligned}
& \Delta_{\mathrm{lh}}=\hbar \omega_{\mathrm{LO}}\left(1+m_{\mathrm{lh}} / m_{\mathrm{e}}\right), \\
& \Delta_{\mathrm{hh}}=\hbar \omega_{\mathrm{LO}}\left(1+m_{\mathrm{hh}} / m_{\mathrm{e}}\right) .
\end{aligned}
$$

$\Delta_{\mathrm{e}}$ is determined by Eq. (1), $m_{\mathrm{lh}}=0.08$ is the light-hole mass and $n=1,2, \ldots$

The full curve in Fig. 2 shows the PLE spectrum of the A sample, illustrating the effect of electron lifetime reduction due to recombination with the holes cascaded to the top of the GaAs valence band. Clear minima in the QW PL intensity appear at the excitation energies, satisfying Eq. (2). Note that the oscillation period $\Delta_{\mathrm{lh}} \approx 2 \Delta_{\mathrm{e}}$. This means that for the excitation energies marked by bars in Fig. 2 electrons and holes return to $k=0$ almost simultaneously and, therefore, have more chance to recombine in the GaAs barrier than to reach the $\mathrm{QW}$. This is confirmed by the analogous oscillations, though opposite in phase (at the energies determined by Eq. (2) the maxima appear), observed in the GaAs barrier PLE spectra.

\section{Conclusions}

The speed and efficiency of carrier transfer into a $\mathrm{QW}$ can be considerably influenced by the surface electric field. Steady-state PLE spectroscopy is shown to be applicable to study the dynamics of photo-excitation transfer into the QW.

\section{References}

[1] B. Deveaud, J. Shah, T.C. Damen, W.T. Tsang, Appl. Phys. Lett.52, 1886 (1988). 
[2] T. Miyoshi, Y. Aoyagi, Y. Segawa, S. Namba, M. Nunoshita, Jpn. J. Appl. Phys. 24, L53 (1985).

[3] G. Bastard, E.E. Mendez, L.L. Chang, L. Esaki, Phys. Rev. B 28, 3241 (1983).

[4] C. Van Hoof, K. Deneffe, J. De Boeck, D.J. Arent, G. Borghs, Appl. Phys. Lett. 54, 608 (1989).

[5] D. Bimberg, J. Christen, A. Steckenborn, G. Weimann, W. Shlapp, J. Lumin. 30, 562 (1985).

[6] G. Ambrazevičius, S. Marcinkevičius, T. Lideikis, K. Naudžius, Semicond. Sci. Technol. 6, 41 (1991).

[7] V. Karpus, unpublished.

[8] S.E. Esipov, Y.B. Levinson, Adv. Phys. 36, 331 (1987). 